\title{
Comparison of safety and efficacy of pregabalin, duloxetine and their combination with epalrestat in diabetic neuropathy: A prospective, double-blind, randomized, controlled Trial
}

\author{
Ravinder Singh ${ }^{1}$, Harbir Kaur Rao ${ }^{2}$, Thakur Gurjeet Singh ${ }^{1 *}$ \\ ${ }^{1}$ Chitkara College of Pharmacy, Chitkara University, Punjab, India. \\ ${ }^{2}$ Gian Sagar Medical College and Hospital, Rajpura, India.
}

\begin{tabular}{l}
\hline ARTICLE INFO \\
\hline Received on: 09/01/2020 \\
Accepted on: 20/08/2020 \\
Available online: $25 / 02 / 2021$ \\
\hline Key words: \\
Diabetes mellitus, neuropathic \\
pain, pregabalin, duloxetine, \\
epalrestat.
\end{tabular}

\begin{abstract}
Neuropathic pain is a common disorder characterized by negative and positive subjective signs and symptoms ranging from numbness to crippling pain. Type 2 diabetes mellitus (T2DM) is the primary cause of neuropathy and neuropathic pain. Diabetic neuropathic pain (DPN) is one of the most common diabetes mellitus complications. The study was aimed to analyze the efficacy and safety of Pregabalin, Duloxetine, and their combination with Epalrestat in T2DM neuropathic patients. The study was conducted on 200 subjects. The patients were divided into 4 groups each comprising of 50 patients. Group I(P) was subjected to Pregabalin (150 mg O.D), Group II (D) to Duloxetine (60 $\mathrm{mg}$ O.D), Group III (P + E) to Pregabalin + Epalrestat (150 mg + $100 \mathrm{mg}$ (O.D), and Group IV (D + E) to Duloxetine + Epalrestat (60 mg + $100 \mathrm{mg}$ (O.D) and) for a period of 6 months. Various clinical parameters like vibration perception threshold, gycated haemoglobin level, visual analog scale, DPNdiabetic diagnostic questionnaire, advance glycated end products, thiobarbituric acid reactive substances, C-reactive proteins, SF12 score, and cost-effectiveness were assessed at baseline and 3 and 6 months. Results demonstrated that Pregabalin and Epalrestat therapy has a better effect on neuropathic pain reduction than Duloxetine and Epalrestat with strict glycemic control and favorably contributes to the health effective benefits by inhibiting disease progression and fulfills the alternate goals of management of DPN. It has been suggested that Pregabalin and Epalrestat therapy is more efficacious and armamentarium for patients with DPN. It has been suggested that Group III therapy is more efficacious, cost-effective, and armamentarium for patients with DPN.
\end{abstract}

\section{INTRODUCTION}

Diabetes mellitus (DM) is a group of metabolic syndrome which is marked by chronic hyperglycemia, glycosuria, hyperlipidemia, and balance of negative $\mathrm{N}_{2}$ (nitrogen) and also sometimes ketonemia resulting from defects in the action of insulin and secretion of insulin, or both which may lead to an impaired mechanism of carbohydrate, lipid, and protein metabolism (Georgoulis et al., 2014; Hossain et al., 2013; Zychowska et al., 2013). The World Health Organization and the Diabetes Data Group classified diabetes as (A) insulin-dependent DM or Type 1 diabetes-idiopathic forms and immune-mediated forms of $\beta$ cell destruction, which results in insulin deficiency. (B) non-insulindependent DM or Type 2 diabetes-disorder to adult-onset, which rises mainly from relative insulin deficiency and insulin resistance. By 2030, diabetics in India is expected to cross 101.2 million and in 20 years' time, expected to rise to 438 million, according to the International Diabetes Federation. About 7 million humans develop DM each year (Hossain et al., 2013). A burden to human health is represented by the increased prevalence of DM due of its longlasting serious microvascular and nacrovascular complications

Abstract of this article was presented at CUDC consortium and Summer School Conference at Chitkara University, India.

*Corresponding Author

Thakur Gurjeet Singh, Chitkara College of Pharmacy, Chitkara

University,Punjab,India.E-mail: gurjeet.singh@chitkara.edu.in 
(Zychowska et al., 2013). Hyperglycemia is the main cause of neuropathic pain and neuropathy. Neuropathyis caused due to both chronic type 1 (T1DM) and type 2 diabetes (T2DM) affecting upto $50 \%$ of those patients suffering from the disease (Zychowska et al., 2013). Neuropathic pain results from the central or peripheral nervous system damage or the disease and is led by a primary lesion or nervous system dysfunctioning. Ironically, about $25 \%$ and $62 \%$ of patients with idiopathic peripheral neuropathy have prediabetes, about $11 \%$ and $25 \%$ are considered to have peripheral neuropathy, and between $13 \%$ and $21 \%$ have neuropathic pain (Georgoulis et al., 2014).

Diabetic neuropathy has multiple symptoms: odd movement with large sensory fibers and frequent cold and/or hot feeling with limited sensory fibers and diagnostic criteria for small fiber neuropathy in clinical practice and research (Grazia et al., 2019). Chronic pain Includes increased perception of pain/response to painful stimuli (hyperalgesia), (allodynia) pain in reaction to stimulus usually not cause pain, (paresthesia) uncomfortable irregular sensation, and random pain. (Colloca et al., 2017; Grazia et al., 2019). Furthermore, the pathophysiology of diabetic neuropathy includes the protein kinase C (PKC) activity, polyol pathway, advanced glycation end products (AGEs), and oxidative stress. Long-term hyperglycemia enhances the polyol pathway and increases nonenzymatic glycation of various structural proteins, which further increases oxidative stress as well as the alteration in the PKC activity and poly-ADP-ribose polymerase activation that are all interrelated for the cause and development of neuropathy. These, in turn, activate or suppress the PKC activity or activate mitogen-activated protein kinase activity, resulting in functional and structural disturbance in the peripheral nervous system (Craige et al., 2016; Veves et al., 2008). Many reasons for neuropathy include lifestyle factors, contaminants to the environment, obesity, cigarette smoking, and nerve tumors. Different types of drugs are also used to treat diabetic neuropathy and neuropathic pain including anticonvulsants, opioids, antidepressants, and aldose reuptake inhibitors (Van Hecke et al., 2014). Antidepressants include tricyclic antidepressants and selective serotonin reuptake inhibitors (SSRI). Tricyclic antidepressants are the first choice in the treatment of neuropathic pain, including painful diabetic neuropathy (Jain et al., 2014). Drugs include amitriptyline, imipramine, desipramine, nortriptyline, maprotiline, and clomipramine. Common side effects include visual blurring, dry mouth, cognitive impairment, tachycardia, orthostatic hypotension, sedation, and weight gain. SSRIs like fluoxetine, venlafaxine, paroxetine, and citalopram have been used for the relief of neuropathic pain. Anticonvulsants are used for the treatment of neuropathic pain. These include phenytoin, carbamazepine, oxcarbazepine, gabapentin, pregabalin, lamotrigine, clonazepam, valproic acid, topiramate, and tiagabine. These agents can be used for the first-line or add-on therapy. Pregabalin binds to the a2-based protein subunit of calcium voltage-gated channels and enhances the release of exciting neurotransmitters. Gabapentin is another drug in the treatment of neuropathic pain (Alles and Smith, 2017; Singh et al., 2016). The flux of glucose is reduced by aldose reductase inhibitors through polyol pathways, impedes the deposition of sorbitol and fructose, and inhibits the reduction of redox potential. It involves tolrestat, zopolrestat, alreastat, and epalrestat (Singh et al., 2016). Pregabalin is one of the generally used therapies currently for a number of neuropathic pain $(\mathrm{NeP})$ conditions (Freynhagen et al., 2015).

A study showed significant decreased pain intensity by both drugs in 66 patients from the Duloxetine group and 77 from the Pregabalin group (Joharchi et al., 2019). Despite the availability of several modalities for the prevention of diabetic neuropathy, mortality and morbidity rates are very high. The present clinical study examined the hypothesis that the combination of Pregabalin or Duloxetine with Epalrestat is efficient in slowing down the development of diabetic neuropathy. Therefore, our study aimed to compare the efficacy and safety of Pregabalin, Duloxetine, and their combination with Epalrestat in T2DM neuropathic patients. The primary outcome of the current clinical study is a decrease in pain score and hindering the progression of disease and secondary outcome measures included quality of life and cost-effectiveness.

\section{MATERIAL AND METHODS}

Permissions to perform the study were acquired from the Institutional Ethics committee (IEC No -PHMA/GSMCH-15/ IEC-38), Gian Sagar Medical College and Hospital, Rajpura, and the study was carried out by adopting the Helsinki agreement and the Effective Medical Practice Code. Patients with DPN having a disease duration of $>10$ years, who are willing to participate in the study and gave written informed consent, were enrolled in the study. Patients were diagnosed on the basis of inclusion and exclusion criteria.

Inclusion and exclusion criteria were designed for the study on the basis of disease duration, Pain Scales, and information from the clinicians.

\section{Inclusion criteria}

1. Patients of either sex with the age of above 18 years.

2. Have pain because of peripheral neuropathy affected by type II diabetes with the pain that starts in the feet and present from at least 6 months assessed by the diagnostic questionnaire (DN4).

3. Might not be pregnant and agree to the use of medically appropriate and effective means of birth control during study participation. Agree to randomize management assignment.

4. May give written informed consent

5. Able to comply with study procedures

\section{Exclusion criteria}

- Severe hepatic disease

- Substance abuse history or dependence within the past year, excluding nicotine and caffeine.

- Unstable cardiovascular or serious, hepatic (acute liver injury such as hepatitis or severe cirrhosis), kidney, respiratory diseases, blood disorder, seizure disorder, problems with peripheral vascular disease, or other medical conditions or psychiatric conditions that would hinder your participation or likely to lead to hospitalization during the course of the study.

- Uncontrolled or poorly controlled hypertension.

All the patients were evaluated for eligibility of inclusion/ exclusion criteria. Clinical evaluation of eligible patients was 
conducted out at baseline and scheduled clinical visits at 3 and 6 months after treatment. At each study visit, including the initial visit, all subjects underwent the same determination. All patients were continuing their previous regimen as such and duloxetine 60 $\mathrm{mg}$, pregabalin $150 \mathrm{mg}$, and epalrestat $100 \mathrm{mg}$ are added according to the groups to which the patient belongs.

\section{STUDY DESIGN}

The study was a double-blind, prospective, randomized, controlled parallel clinical study and conducted out in the Outpatient department, based at Gian Sagar Medical College and Hospital, Rajpura. The study lasted from November 2015 to January 2017. Clinical assessment of eligible patients was carried out at baseline and scheduled clinical visits including visual analog scale (VAS), Vibration Perception Threshold (VPT), DN4, gycated haemoglobin (HbA1c), thiobarbituric acid reactive substances (TBARS), AGEs, C-reactive proteins (CRP), and SF 12 at 3 and 6 months after the involvement of biochemistry parameters in the study using enzyme- linked immunosorbent assay analytical kits. At each study visit, including the initial visit, all subjects underwent the same determination.

Groups: The patients will be randomly divided into four groups and by appropriate sample size analysis of each group consisting of 200 patients, and coding of investigational product is accordingly done for double-blind study by following the Inclusion and Exclusion criteria:

Group I: Duloxetine; $60 \mathrm{mg} /$ days (D), Group II: Pregabalin; 150 mg/days (P), Group III: Pregabalin 150 mg/days + Epalrestat 100 mg/days (P + E); Group IV: Duloxetine 60 mg/ days + Epalrestat $100 \mathrm{mg} /$ days $(\mathbf{D}+\mathbf{E})$.

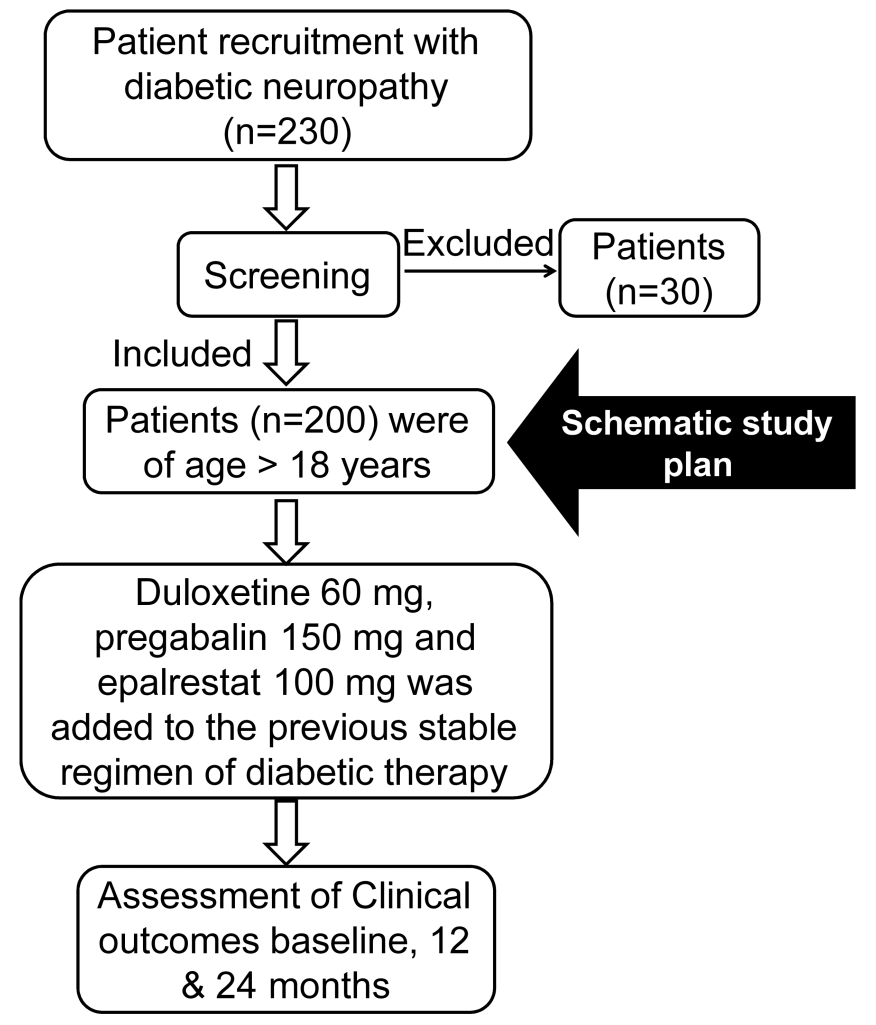

Schematic study plan
All the data were collected and analyzed at scheduled clinical visits using the following parameters: Glycated hemoglobin: the 2009 International Expert Committee advised the usage of HbAlc to diagnose diabetes with a threshold of $6.5 \%$ (Edwards et al., 2008). C-reactive protein: high levels of serum CRP in a normal population is a sign of future development of diabetes. The levels of CRP in blood correlate with the severity of diabetes and the level of control (Farmer et al., 2012). AGE are developed from the overload of proteins, lipids, and nucleotides by the nonenzymatic reaction of glucose, which results in axon and nerve cell metabolism intervention and hence inhibits neuronal integrity and repair mechanisms (Yagihashi et al., 2007). VPT is a tool to detect diabetic neuropathy and dysfunction of nerves (Vinik, 2010).

Visual Analog scale (VAS): The VAS is a measure of pain intensity. VAS is a scale of $10 \mathrm{~cm}(100 \mathrm{~mm})$ in length. For pain intensity, the scale is utmost generally anchored by "no pain" (score of 0) and "worst imaginable pain" (score of 10) (Koltezenburg and Scadding, 2001). DN4 Questionnaire (DN4 Q): Diabetic neuropathic pain (DPN) will be evaluated by administering the DN4 Q. This questionnaire consists of a set of four questions for the assessment of neuropathic pain (Khwaja and Chaudhry, 2007). TBARS: The excess production of free radicals in diabetes can trigger diabetic neuropathy via many mechanisms occurring in both the central and peripheral nervous systems. It is seen that this is the source of disorders of the nervous system (Bansal et al., 2006). Cost-effective analysis: Cost-Effectiveness Analysis contains the comparison of programs or management substitutes with different safety and efficacy profiles. Cost is calculated in rupees, and tests are also represented in units of effectiveness, a normal unit, or nonrupees (Syed, 2011).

Quality of Life: The U.S. Food and Drug Administration (FDA) has been boosting the use of Patient-Reported Outcomes in clinical studies to help offer materials regarding the status of the impact of the disease on patients' mental and physical health (Pradhan et al., 2001).

\section{Statistical analysis}

Continuous data are shown as mean \pm standard error of the mean. An evaluation between the groups was done using unpaired student t-tests. Statistical significance was assumed at $p<0.05$. Statistical analysis was performed using Sigmastat 4.0 (Systat Software, San Jose, CA, USA).

\section{RESULTS}

Overall, 200 patients were involved in the study according to the exclusion and inclusion criteria. The study was followed, and the response was measured at baseline (0 days), 3 m,and 6 months.

\section{Effect of treatments on glycated hemoglobin (\%) (HbA1c)}

Duloxetine + epalrestat management for a time span of 3 and 6 months created a difference from baseline of $11.586 \pm$ 0.183 to $8.836 \pm 0.187$ in 3 months and $6.22 \pm 0.162$ in 6 months. With duloxetine, glycated hemoglobin was from $11.126 \pm 0.258$ to $10.06 \pm 0.229$ in 3 months and $9.092 \pm 0.215$ in 6 months. The decrease in HbA1c for the duloxetine + epalrestat group of patients was statistically significant relative to the respective 
baseline value $(p<0.05)$. Pregabalin + Epalrestat treatment for a period of 3 and 6 months produced a difference from baseline of $12.116 \pm 0.168$ to $9.57 \pm 0.155$ in 3 months and $7.24 \pm 0.118$ in 6 months. With pregabalin, the change was from $11.094 \pm 0.239$ to $9.69 \pm 0.228$ in 3 months and $8.394 \pm 0.215$ in 6 months. The lessening in $\mathrm{HbA} 1 \mathrm{c}$ in the pregabalin + Epalrestat group equated to the relevant baseline values was statistically significant $(p<0.05)$. $\mathrm{HbA} 1 \mathrm{c}$ for $\mathrm{D}+\mathrm{E}$ and $\mathrm{P}+\mathrm{E}$ for 3 and 6 months and Duloxetine + epalrestat treatment for a period of 3 and 6 months produced a difference from $8.836 \pm 0.187$ in 3 months and $6.22 \pm 0.162$ in 6 months, respectively (Fig. 1).

\section{Effect of treatments on AGEs $(\mu \mathrm{g} / \mathrm{ml})$}

Pregabalin + Epalrestat and Pregabalin treatment for a time of 3 and 6 months formed a reduction in AGE from baseline of $4.28 \pm 0.32$ to $3.05 \pm 0.29$ in 3 months and $2.1 \pm 0.15$ in 6 months with Pregabalin + Epalrestat and the lessening in AGEs by pregabalin from baseline $4.33 \pm 0.34$ to $3.75 \pm 0.23$ in 3 months and $3.3 \pm 0.21$ in 6 months. The decrease in AGEs in the pregabalin + epalrestat group compared to the respective baseline values was statistically significant $(p<0.05)$. Duloxetine + epalrestat treatment for a period of 3 and 6 months produced a difference from baseline of $4.2 \pm 0.34$ to $3.3 \pm 0.31$ in 3 months and $2.9 \pm$ 0.271 in 6 months, with duloxetine from $4.3 \pm 0.387$ to $3.9 \pm 0.27$ in 3 months and $3.6 \pm 0.21$ in 6 months. The decrease in AGEs in the duloxetine + epalrestat group compared to the respective baseline value was statistically significant ( $p<0.05$; Fig. 2$)$.

\section{Effect of managements on CRP- (g/dl)}

Duloxetine + epalrestat management for a time span of 3 and 6 months produced the difference from baseline of $8.544 \pm$ 0.168 to $6.43 \pm 0.14$ in 3 months and $4.2 \pm 0.069$ in 6 months with duloxetine and the decrease in inflammation score by duloxetine from baseline $8.178 \pm 0.202$ to $7.164 \pm 0.207$ in 3 months and $6.182 \pm 0.21$ in 6 months (Fig. 5a and b). The decrease in CRP in the duloxetine + epalrestat group compared to their respective

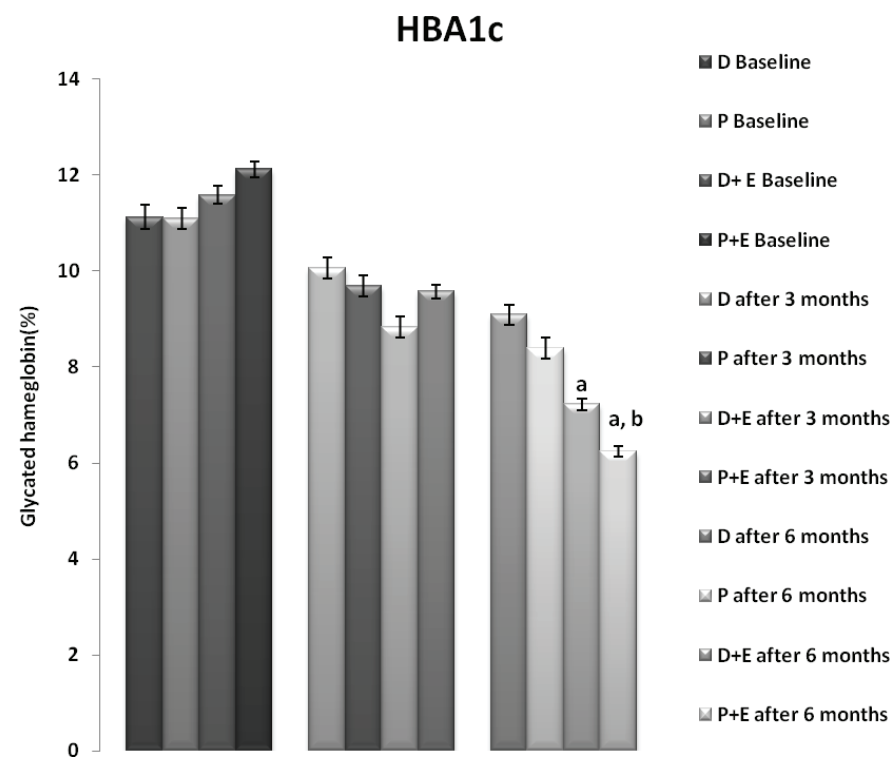

Figure 1. Effect of various treatments on HbA1c in Diabetic Neuropathic Patients. baseline value was statistically significant $(p<0.05)$. Pregabalin + Epalrestat and Pregabalin management for a time span of 3 and 6 months produced a decrease in CRP from baseline of $8.308 \pm$ 0.29 to $6.23 \pm 0.22$ in 3 months and $3.9 \pm 0.09$ in 6 months with Pregabalin + Epalrestat and the lessening in CRP by pregabalin from baseline of $7.772 \pm 0.331$ to $6.342 \pm 0.221$ in 3 months and $5.11 \pm 0.111$ in 6 months. The decrease in CRP in the Pregabalin + Epalrestat treated patients compared to a particular baseline value was statistically significant $(p<0.05)$ (Fig. 3).

\section{Effect of managements on VPT}

Duloxetine + epalrestat treatment for a period of 3 and 6 months produced a difference from baseline of $30.4 \pm 0.27$ to $27.6 \pm 0.22$ in 3 months and $24.8 \pm 0.12$ in 6 months and with duloxetine from baseline of $29.2 \pm 0.19$ to $26.5 \pm 0.16$ on 3 months and $23.8 \pm 0.13$ in 6 months. Pregabalin + Epalrestat treatment for a period of 3 and 6 months produced the difference from baseline of $29.1 \pm 0.481$ to $26.6 \pm 0.481$ in 3 months and $24.8 \pm 0.481$ in 6 months and with pregabalin from baseline of $27.2 \pm 0.21$ to 25.1 \pm 0.19 in 3 months and $24.6 \pm 0.23$ in 6 months. Comparative analysis of all groups on VPT has shown no statistically significant change (Fig. 4).

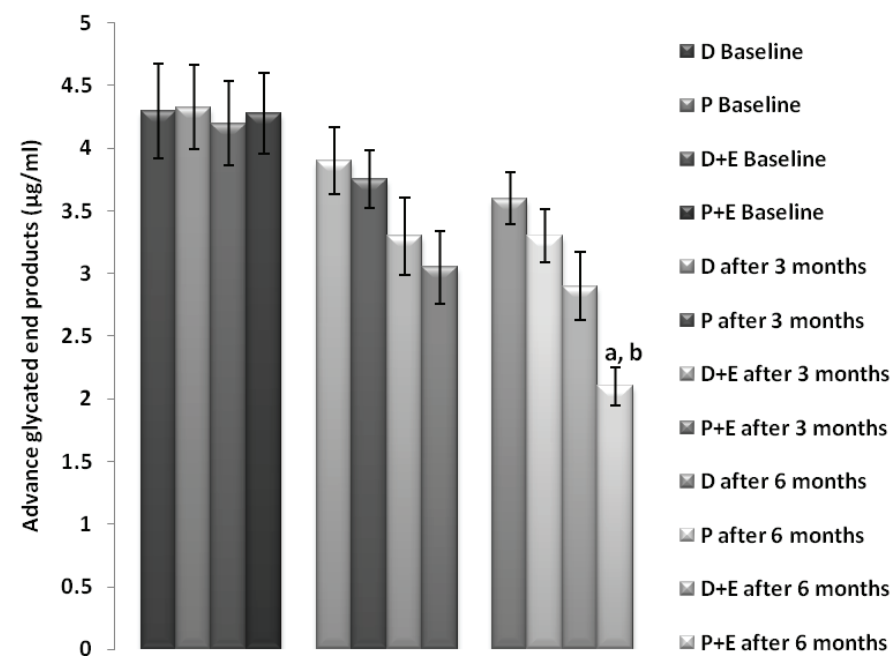

Figure 2. Effect of various treatments on AGEs in Diabetic Neuropathic Patients.

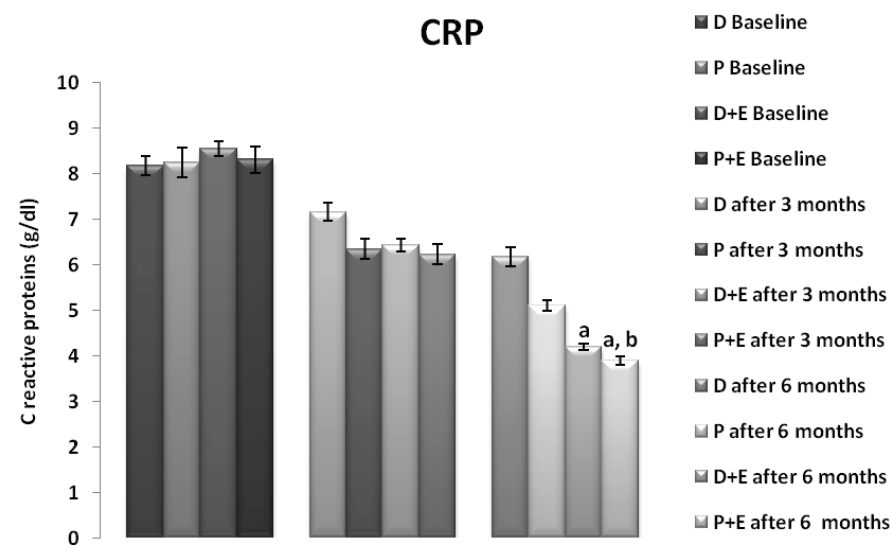

Figure 3. Effect of various treatments on CRP in Diabetic Neuropathic Patients 


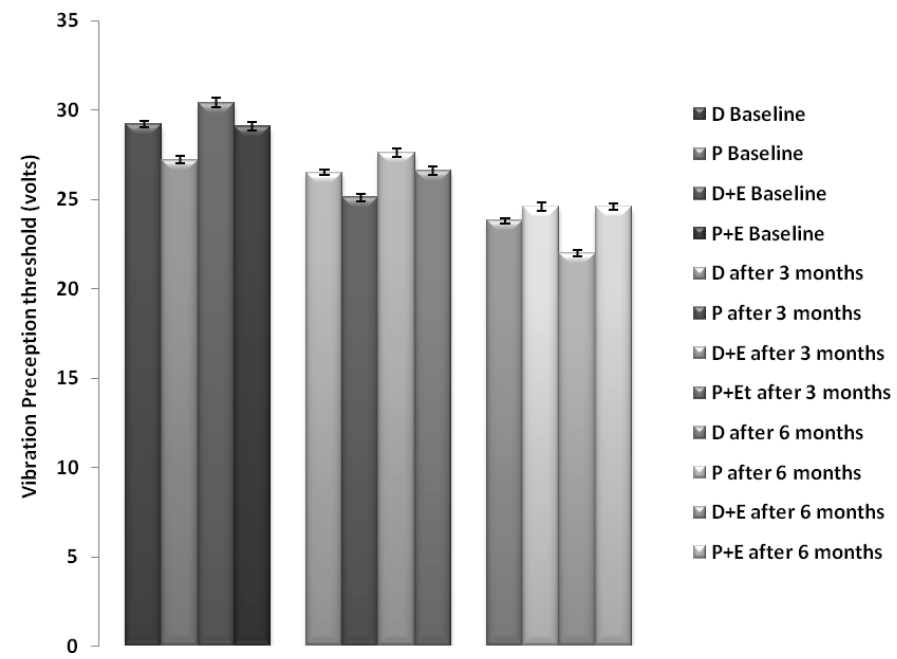

Figure 4. Effect of various treatments on VPT in Diabetic Neuropathic Patients.

\section{Effect of managements on the VAS}

Duloxetine + epalrestat management for a time span of 3 and 6 months produced the difference from baseline of $8.42 \pm$ 0.27 to $5.08 \pm 0.22$ in 3 months and $3.9 \pm 0.17$ in 6 months and with Duloxetine from baseline of $8.86 \pm 0.12$ to $6.8 \pm 0.16$ in 3 months and $4.6 \pm 0.13$ in 6 months. The decrease in VAS score in the Duloxetine + epalrestat and duloxetine group compared to respective baseline values was statistically significant $(p<0.5)$. Pregabalin + Epalrestat treatment for a period of 3 and 6 months produced a difference from baseline of $8.46 \pm 0.24$ to $4.4 \pm 0.21$ in 3 months and $2.96 \pm 0.18$ in 6 months and with Pregabalin from baseline of $8.74 \pm 0.21$ to $5.2 \pm 0.019$ in 3 months and $4.1 \pm 0.023$ in 6 months. The lessening in VAS pain parameter in the pregabalin + epalrestat and pregabalin treated group compared to the relevant baseline value was statistically significant ( $p<0.05$; Fig. 5).

\section{Effect on DN4 Q}

Duloxetine + epalrestat management for a time span of 3 and 6 months produced the difference from baseline of $8.42 \pm$ 0.27 to $4.1 \pm 0.22$ in 3 months and $2.9 \pm 0.17$ in 6 months and with Duloxetine from baseline of $7.8 \pm 0.19$ to $5.7 \pm 0.016$ in 3 months and $4.2 \pm 0.13$ in 6 months. The reduction in the DN4 score in the duloxetine + epalrestat group compared to the specific baseline values was statistically significant, i.e., $p<0.05$. Pregabalin + Epalrestat treatment for a period of 3 and 6 months produced a difference from baseline of $8.32 \pm 0.24$ to $4.9 \pm 0.21$ in 3 months and $3.5 \pm 0.018$ in 6 months and with Pregabalin from baseline of $8.2 \pm 0.021$ to $4.66 \pm 0.19$ in 3 months and $3.4 \pm 0.23$ in 6 months. The reduction in the DN4 score in the pregabalin + epalrestat and pregabalin group equated to the respective baseline value was statistically significant ( $p<0.05$; Fig. 6).

\section{Effect of treatments on TBARS}

Duloxetine + epalrestat management for a period of 3 and 6 months produced the difference from baseline of $6.42 \pm$ 0.172 to $4.2 \pm 0.152$ in 3 months and $3.6 \pm 0.109$ in 6 months and with Duloxetine from baseline of $6.54 \pm 0.31$ to $5.2 \pm 0.25$ on 3 months and $4.22 \pm 0.23$ in 6 months respectively. Pregabalin + Epalrestat treatment for a period of 3 and 6 months produced the

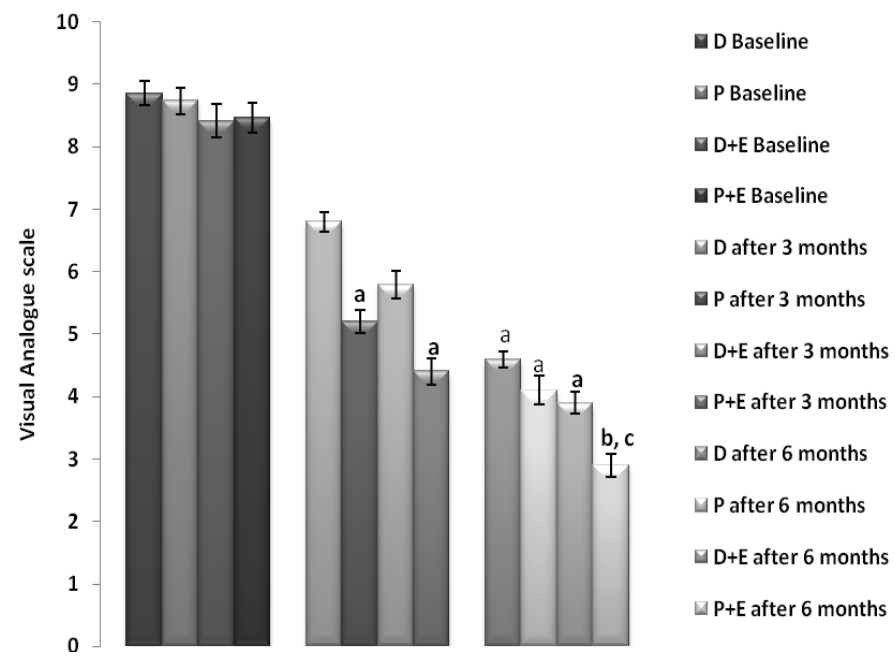

Figure 5. Effect of various treatments on VAS in Diabetic Neuropathic Patients.

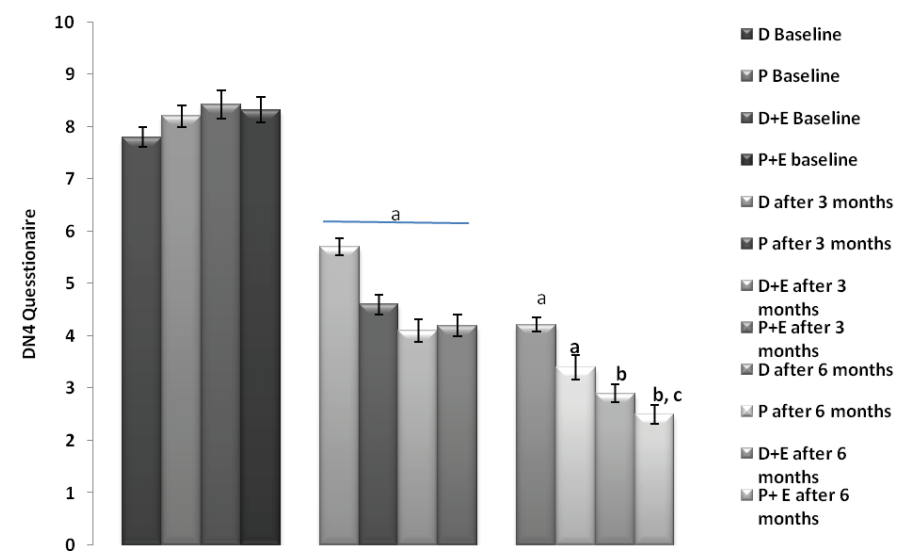

Figure 6. Effect of various treatments on DN4 Q in Diabetic Neuropathic Patients.

difference from baseline of $6.32 \pm 0.172$ to $3.9 \pm 0.154$ in 3 months and $2.7 \pm 0.0993$ in 6 months and with Pregabalin from baseline of $6.8 \pm 0.28$ to $5.66 \pm 0.272$ in 3 months and $4.12 \pm 0.263$ in 6 months. The decrease in TBARS in treatment groups equated to their individual baseline assessment was statistically significant ( $p$ $<0.05$; Fig. 7).

\section{Effect of treatments on SF 12 physical health score (PCS)}

Duloxetine + epalrestat management for a phase of 6 months produced the difference from baseline of $37.54 \pm 1.82$ and $56.12 \pm 2.2$ in 6 months and with Duloxetine from baseline of 37.33 \pm 1.9 to $53.22 \pm 2.38$ in 6 months. Pregabalin + Epalrestat treatment for a period of 6 months produced the difference from baseline of $38.23 \pm 2.09$ and $65.33 \pm 2.46$ in 6 months and with Pregabalin from baseline of $39.23 \pm 1.33$ to $58.57 \pm 2.01$ in 6 months. The reduction in PCS in the studied groups compared to the respective baseline value was statistically significant ( $p<0.05$; Fig. 8).

\section{Effect of treatments on SF 12 mental health score (MCS)}

Duloxetine + epalrestat management for a phase of 6 months showed the difference from baseline of $42.23 \pm 1.92$ and $61.12 \pm 2.62$ in 6 months and with Duloxetine from baseline of 


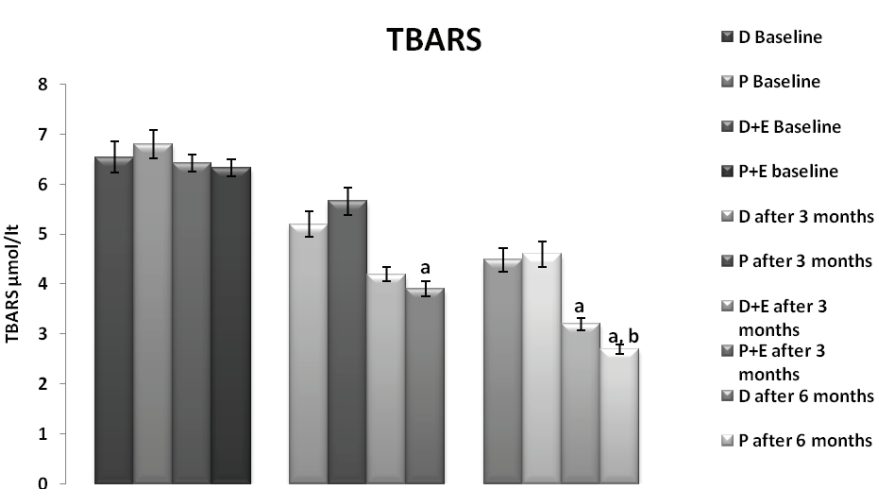

Figure 7. Effect of various treatments on TBARS in Diabetic Neuropathic Patients.

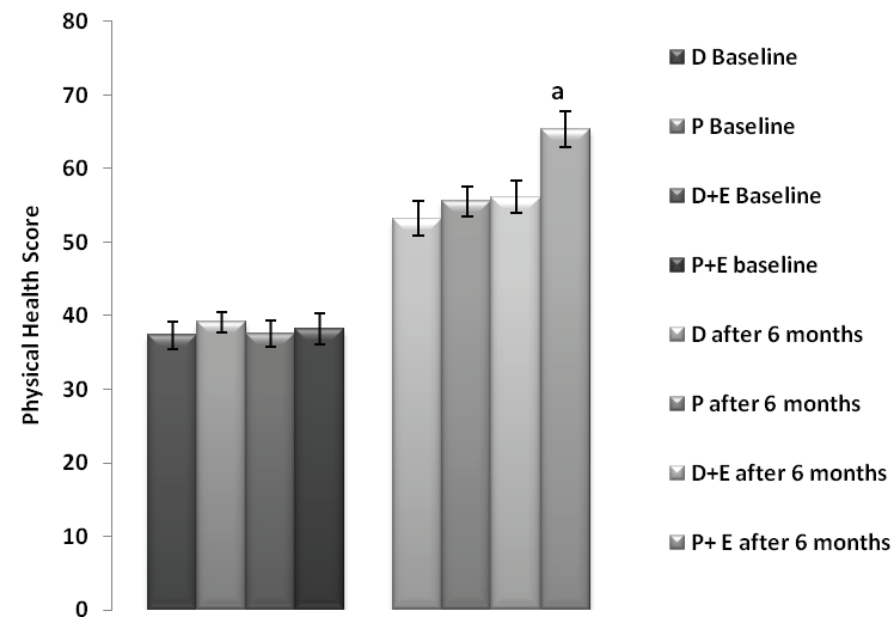

Figure 8. Effect of various treatments on PHS in in Diabetic Neuropathic Patients.

$42.33 \pm 1.55$ to $63.32 \pm 2.8$ in 6 months. Pregabalin + Epalrestat treatment for a period of 6 months produced the difference from baseline of $44.13 \pm 2.15$ and $58.31 \pm 2.59$ in 6 months and with Pregabalin from baseline of $43.13 \pm 1.56$ to $58.57 \pm 2.3$ in 6 months. The decrease in MCS in both groups compared to their particular baseline assessment was statistically significant $(p<$ 0.05; Fig. 9).

\section{Cost-effectiveness}

From the present study, the pharmacoeconomics evaluation shows that $\mathrm{P}+\mathrm{E}$ treatment is cost-effective in comparison to the $\mathrm{D}+\mathrm{E}$ treatment concerning for direct cost and incremental cost effective ratio (ICER). The ICER for $\mathrm{P}+\mathrm{E}$ treatment works out to be 145.16 rupees (Tables 1 and 2), which means that it costs 145.16 rupees to generate each additional unit of health benefit gained in adding $\mathrm{P}+\mathrm{E}$ to the existing medical regimen (percentage change in effect with $\mathrm{P}+\mathrm{E}$ is $53.90 \%$ and $47.70 \%$ with D + E concerning for cost of treatment; Fig. 10).

\section{DISCUSSION}

Diabetic peripheral neuropathy directly affects the physical functioning of patients and become disabled. It is highly prevalent. Painful diabetic peripheral neuropathy is a challenging

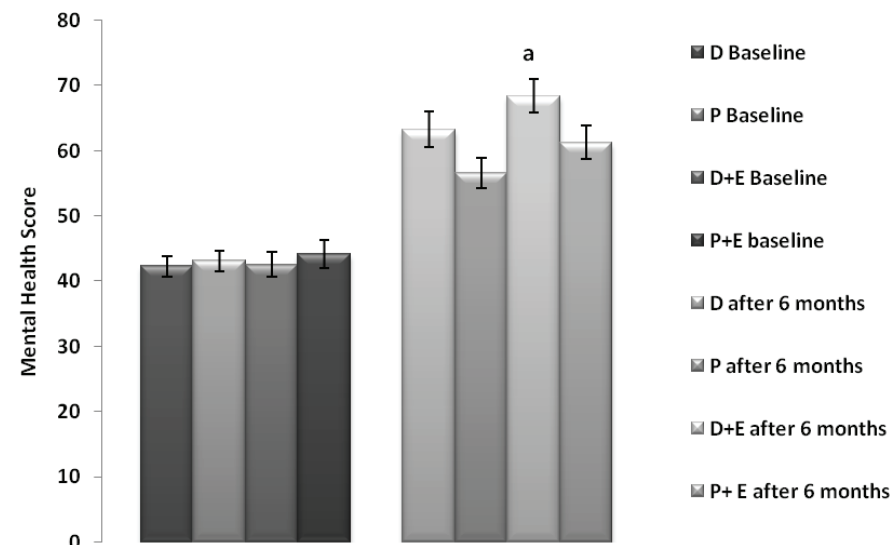

Figure 9. Effect of various treatments on MHS in Diabetic Neuropathic Patients.

Table 1. Incremental cost effective analysis in diabetic neuropathy patients.

\begin{tabular}{lcc}
\hline Treatment & 24 weeks cost per patient & ICER*/month \\
\hline Pregabalin + Epalristat GpIII & $5,670 \mathrm{Rs}$ & $145.16 \mathrm{Rs}$. \\
Duloxitene + Epalristat & $4,770 \mathrm{Rs}$ & - \\
Gp IV & & \\
\hline
\end{tabular}

The average total cost of $\mathrm{P}+\mathrm{E}$ versus $\mathrm{D}+\mathrm{E}$ treated group for 24 weeks was found to be Indian Rs. 5,670 and 4,770, respectively.

neuropathic pain syndrome (Syed, 2011). Pain management is an essential component in the comprehensive care of diabetic patients. Neuropathy is frequently linked with important burning, stabbing, or tingling pain and numbness, and may show sleep interference, depression, anxiety, and severe disability as a consequence. In the present time, only glucose control and pain management like treatments are effective, but they do not prevent nerve degeneration (Pradhan et al., 2001). High levels of proof help the usage of evident anticonvulsants and antidepressants for the management of pain in diabetic peripheral neuropathy, opioid, topical agents, and $\alpha-2 \delta$ ligands. However, two drugs, Duloxetine and Pregabalin, have received specific FDA approval for the treatment of diabetic peripheral neuropathic pain (DPNP) (Ryle and Donaghy, 1995). In addition to epalrestat, the AGE inhibitor is a widely used drug in diabetic complications. Overall, it is assessed that diabetic peripheral neuropathy (DNP) progresses in 10\%-20\% of the diabetic population and may be found in $40 \%-60 \%$ with documented (Ferraz et al., 1990; Garrow and Boulton, 2006). One research revealed that approximately $12 \%$ of patients with DNP had not ever stated this disorder to their clinicians (Bouhassira et al., 2005). Glucose control decreases the progression of the disease. In 1993, the DCCT study group tracked over 1,400 subjects for 5 years and discovered a $60 \%$ lessening in the development of neuropathy in maintaining strict glycemic control. In a study for 3 months, the randomized, double-blind multicenter research evaluated the efficacy of Pregabalin in the management of painful diabetic neuropathy. A total of 246 men and women having painful diabetic neuropathy received Pregabalin (150 or $600 \mathrm{mg} /$ day) or placebo. The results of the efficacy of drugs reported that Pregabalin $600 \mathrm{mg} /$ days significantly decreased the mean pain score to 4.3 versus 5.6 for placebo. More patients receiving Pregabalin $600 \mathrm{mg}$ /days showed an improvement, as rated on the 
Table 2. Contribution of various parameters in direct medical cost in DPN.

\begin{tabular}{|c|c|c|c|c|c|}
\hline S. No & Direct cost & Description & $\begin{array}{c}\text { Average cost (duloxitene }+ \\
\text { epalristat) per patient }\end{array}$ & Description & $\begin{array}{c}\text { Average cost in Rupee per } \\
\text { patient (Pregabalin + Epalristat) }\end{array}$ \\
\hline \multirow[t]{4}{*}{1} & Cost of medicine & Duloxetine & $525^{\mathrm{a}}$ Rs. & Pregabalin & $675 \mathrm{Rs}$ \\
\hline & & Epalristat & $270^{\mathrm{b}}$ Rs. & Epalristat & $270 \mathrm{Rs}$ \\
\hline & & Oral Hypoglycemics & $180.00^{\mathrm{c}}$ Rs. & Oral Hypoglycemics & $180 \mathrm{Rs}$ \\
\hline & & Other drugs & $155.44^{\mathrm{d}}$ Rs. & Other drugs & 155.44 Rs \\
\hline 2 & Monitoring cost & Lab & $83.33^{\mathrm{e}}$ Rs. & Monitoring cost & 83.33Rs \\
\hline 3 & Consultation Charges & Doctor consultation & $5^{\mathrm{f}} \mathrm{Rs}$. & Doctor consultation & $5 \mathrm{Rs}$ \\
\hline \multicolumn{2}{|c|}{ Total cost } & & $1,135.44$ & & $1,280.44$ \\
\hline
\end{tabular}

Average cost of treatment for 1 month has been shown in Indian Rs.

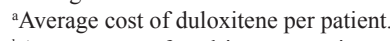

${ }^{\mathrm{b}}$ Average cost of epalristat per patient.

${ }^{\mathrm{c}}$ Average cost of oral hypoglycemic agents.

${ }^{\mathrm{d}}$ Average cost other adjuvant drugs like PPIs \& multivitamins.

${ }^{\mathrm{e} A v e r a g e ~ l a b ~ p a r a m e t e r s ~ c o s t ~ d u r i n g ~} 3$ phases of study per patient.

\section{$\%$ Change Effect}

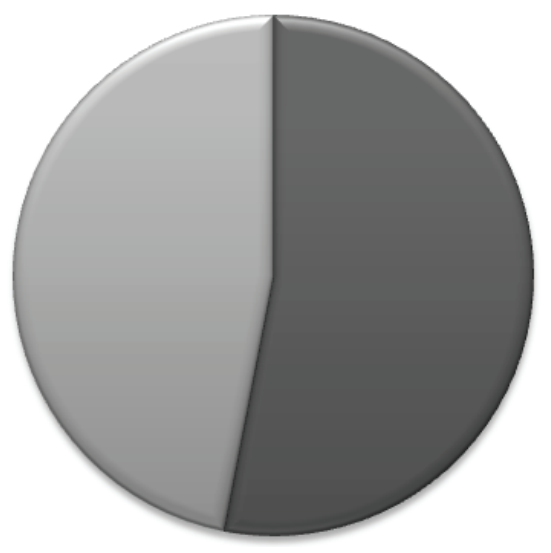

Figure 10. Percentage change in effect with $\mathrm{P}+\mathrm{E}$ and $\mathrm{D}+\mathrm{E}$ with respect to cost of treatment in Diabetic Neuropathic Patients.

Clinical and Patient Global Impression of Change Scales, 73\% vsversus $45 \%, 85 \%$ vsversus $47 \%$, than placebo respectively. The study results showed that Pregabalin $600 \mathrm{mg} /$ days was safe and effective in reducing the pain and other associated symptoms of painful diabetic neuropathy (Kitto et al., 1992). Duloxetine $60 \mathrm{mg} /$ days proved statistically significantly larger progress compared with placebo on the pain score, through the 12-weeks trial. Duloxetine at $60 \mathrm{mg}$ /days and $120 \mathrm{mg} /$ days was safe and efficient in the treatment of diabetic peripheral neuropathic pain (Callaghan et al., 2012; Detsky and Naglie, 1990; Sundaram et al., 2007). Data collected from six clinical trials were assessed, and it was observed that Epalrestat $50 \mathrm{mg} 3$ times/days may enhance subjective neuropathy symptoms and motor and sensory nerve conduction velocity (Charles et al., 2006) Some studies have shown that Pregabalin, Epalrestat, and Duloxetine as individual drugs were used to show the clinically significant decrease in pain earlier (Daousi et al., 2004; Richter et al., 2005). However, uptil now, there has been no research which directly compares the effect of Duloxetine, Pregabalin, and Epalrestat in the case of DPN. The present study was conducted to compare the efficacy and safety of two drugs, i.e., Duloxetine and Pregabalin with the combination of
Epalrestat on neuropathic pain in diabetic patients which was the double-blind clinical study to prevent biased results. The DoubleBlind Clinical Study is a clinical trial in which the subjects and the researchers were not knowing which active medication, treatment, etc., they are receiving and which subjects were not receiving: it is one of the methods for excluding subjective bias from the test results. The finest and most dependable form of research is doubleblind. The purpose of this type of study is to eradicate the power of suggestion (Goldstein et al., 2005; Raskin et al., 2005; Ramirez and Borja, 2008). The double-blind study retains both doctors and participants in the dark as to who is receiving which treatment. The double-blind procedure is a method of enhancing internal validity in an experiment. The outcome of the present study revealed a significant decrease in pain score, HbAlc, CRP, AGE, TBARS, and SF 12 score with the combination of Pregabalin + Epalrestat and Duloxetine + Epalrestat in comparison to duloxetine and pregabalin alone. This reflects that when epalrestat was given in combination with pregabalin and duloxetine, there is a greater reduction in pain, $\mathrm{HbA1c}$, inflammation, AGEs, and SF12 score with glycemic control in patients with DPNP. The combination of pregabalin + epalrestat is cost-effective in comparison to duloxetine + epalrestat and showed better therapeutic results. Epalrestat, in hyperglycemia, reduces intracellular sorbitol accumulation by an uncompetitive aldose reductase inhibition, reducing the progression of symptoms, which shows that Epalrestat is more efficacious when used in combination with oral hypoglycemic agents. Pregabalin interacts with $\alpha-2 \delta$ subunit of voltage-gated calcium channels in the presynaptic neurons and reduces the entry of calcium causing a drop in the release of many excitatory neurotransmitters, i.e., Glutamate, Substance P, CGRP, and Noradrenaline, leads to the reduction of the pain intensity and Duloxetine works by inhibiting serotonin and norepinephrine reuptake which works as inhibitors for pain impulses transmission (Lesser et al., 2004; Rosenstock et al., 2004). The probable intention for better efficacy of Epalrestat is because of its lowering of intracellular sorbitol accumulation. The adverse effects were observed in $6 \%(3 / 50)$ of patients in the group treated with Pregabalin and $8 \%(4 / 50)$ of patients in the groups treated with Duloxetine. Patients taking Pregabalin therapy faced dizziness with $10 \%(n=5)$, dry mouth with $5 \%(n=1)$, weight gain was combated in two patient $4 \%(n=2)$. In the Duloxetine treated 
group, two patients experienced somnolence $6 \%(n=3)$, dry mouth $5 \%(n=1)$, constipation was encountered in one patient $(5 \%)$, and nausea observed in two patients (4\%). In the epalrestat treated group, two patients experienced g.i.t discomfort 5\% $(n=1)$, nausea, and vomiting $10 \%(n=5)$. In the present study, to assess the safety of Pregabalin, Duloxetine, and Epalrestat, evaluation of biochemical parameters disclosed no significant changes in the patients. However, in the duloxetine-treated group, a minor decrease in blood pressure and heart rate was detected, but it was of no significance. All other safety parameters, namely, serum creatinine, total leucocyte count, hemoglobin, differential leucocytes count, erythrocyte sedimentation rate, aspartate aminotransferase, and alanine aminotransferase, did not indicate any significant change.

The drug therapies were discovered to be well tolerated. Any major adverse effects were not observed throughout the course of the research in the treated groups, i.e., during the 6-month duration. Pregabalin showed minor side effects like dry mouth, dizziness, and weight gain, whereas Duloxetine reported dry mouth, nausea, constipation, and gastrointestinal tract discomfort with Epalrestat. Based on the results obtained in the present study, it has been determined that duloxetine and pregabalin are efficient in the decrease of DPN but pregabalin is more potent and efficacious in decreasing neuropathic pain probably by restraining the release of neurotransmitters concerned in transmission of the pain signal. Pregabalin + Epalrestat showed a major reduction in the VAS and DN4 Q as compared to Duloxetine + Epalrestat. Epalrestat was able to hinder the progression of neuropathy and stops further nerve degeneration with strict glycemic control. Pharmacoeconomic evaluation revealed that the combination of Pregabalin + Epalrestat is cost-effective in comparison to Duloxetine + epalrestat and showed better therapeutic results. Hence, we can conclude from this current study that Pregabalin + Epalrestat treatment has enhanced effectiveness intended for decreasing neuropathic pain than Duloxetine + epalrestat with strict glycemic control.

\section{CONCLUSION}

From the present study, it is concluded that the treatment with Pregabalin + Epalrestat favorably contributes to the effective health benefits by inhibiting disease progression and fulfills the alternate goals of management of DPN. The results of the present study conclude that Pregabalin + Epalrestat treatment is more efficacious, cost-effective, and armamentarium for patients with DPN.

\section{ACKNOWLEDGMENTS}

The authors are grateful to the Chitkara College of Pharmacy, Chitkara University, Rajpura, Patiala, Punjab, India, and Gian Sagar Medical College, and Hospital Rajpura for providing the necessary facilities to carry out the research work.

\section{AUTHOR CONTRIBUTIONS}

All authors made substantial contributions to conception and design, acquisition of data, or analysis and interpretation of data; took part in drafting the article or revising it critically for important intellectual content; agreed to submit to the current journal; gave final approval of the version to be published; and agree to be accountable for all aspects of the work. All the authors are eligible to be an author as per the international committee of medical journal editors (ICMJE) requirements/guidelines.

\section{FUNDING}

There is no funding to report.

\section{CONFLICTS OF INTEREST}

The authors report no financial or any other conflicts of interest in this work.

\section{PUBLISHER'S NOTE}

This journal remains neutral with regard to jurisdictional claims in published institutional affiliation.

\section{REFERENCES}

Alles SR, Smith PA. The anti-allodynic gabapentinoids: myths, paradoxes, and acute effects. The Neuroscientist. , 2017; 23(1):40-55.

Argoff CE, Cole BE, Fishbain DA, Irving GA. Diabetic peripheral neuropathic pain: clinical and quality-of-life issues. Mayo Clin Proc, 2006; 81(4):S3-S11.

Bansal V, Kalita J, Misra UK. Diabetic neuropathy. Postgrad Med J, 2006; 82(964):95-100.

Bouhassira D, Attal N, Alchaar H, Boureau F, Brochet B, Bruxelle J, Cunin G, Fermanian J, Ginies P, Grun-Overdyking A, JafariSchluep H. Comparison of pain syndromes associated with nervous or somatic lesions and development of a new neuropathic pain diagnostic questionnaire (DN4). Pain, 2005; 114(1-2):29-36.

Callaghan BC, Cheng HT, Stables CL, Smith AL, Feldman EL. Diabetic neuropathy: clinical manifestations and current treatments. Lancet Neurol, 2012; 11(6):521-34.

Charles EA, Cole BE, Fishbain DA, Irving GA. Diabetic peripheral neuropathic pain: clinical and quality-of-life issues. Mayo Clin Proc, 2006; 81(4):S3-11.

Colloca L, Ludman T, Bouhassira D, Baron R, Dickenson AH, Yarnitsky D, Freeman R, Truini A, Attal N, Finnerup NB, Eccleston C. Neuropathic pain. Nature rReviews Disease pPrimers. , 2017; 3(1):1-9.

Craige SM, Reif MM, Kant S. Mixed-lineage protein kinases (MLKs) in inflammation, metabolism, and other disease states, Biochimica et Biophysica Acta (BBA)-Molecular Basis of Disease., 2016; 1862(9):1581-6.

Daousi C, MacFarlane IA, Woodward A, Nurmikko TJ, Bundred PE, Benbow SJ. Chronic painful peripheral neuropathy in an urban community: a controlled comparison of people with and without diabetes. Diabet Med, 2004; 21(9):976-82.

Detsky AS, Naglie IG. A clinician's guide to cost-effectiveness analysis. Ann Intern Med, 1990; 113(2):147-54.

Edwards JL, Vincent AM, Cheng HT, Feldman EL. Diabetic neuropathy: mechanisms to management. Clin Pharmacol Ther, 2008; 120(1):1-34.

Farmer AJ, Perera R, Ward A, Heneghan C, Oke J, Barnett AH, Davidson MB, Guerci B, Coates V, Schwedes U, O’Malley S. Metaanalysis of individual patient data in randomised trials of self monitoring of blood glucose in people with non-insulin treated type 2 diabetes. BMJ, 2012; 27:344-486.

Ferraz MB, Quaresma MR, Aquino LR, Atra E, Tugwell P, Goldsmith $\mathrm{CH}$. Reliability of pain scales in the assessment of literate and illiterate patients with rheumatoid arthritis. J Rheumatol, 1990; 17:1022-4.

Freynhagen R, Serpell M, Emir B, Whalen E, Parsons B, Clair A, Latymer M. A comprehensive drug safety evaluation of pregabalin in peripheral neuropathic pain. Pain Practice., 2015; 15(1):47-57.

Garrow AP, Boulton AJ. Vibration perception threshold-a valuable assessment of neural dysfunction in people with diabetes. Diabetes Metab Res Rev, 2006; 22(5):411-9. 
Georgoulis M, Kontogianni MD, Yiannakouris N. Mediterranean diet and diabetes: prevention and treatment. Nutrients, 2014; 6(4):1406-23.

Goldstein DJ, Lu Y, Detke MJ, Lee TC, Iyengar S. Duloxetine vs. placebo in patients with painful diabetic neuropathy. Pain, 2005; 116(12):109-18.

Grazia D, Sara R, Raffaella L, Daniele C, Margherita M, Erika S, Roberto E, Giuseppe L. Diagnostic criteria for small fibre neuropathy in clinical practice and research. Brain, 2019; 142(12):3728-36.

Hossain MM, Shoaib SM, Mallick R, Hore R, Tanmy TT. An assessment of present status and related complications of Diabetes Mellitus (DM) at port city Chittagong in Bangladesh. Stand Res J Med Med Sci, 2013; 1:6-11.

Jain D, Bansal MK, Dalvi R, Upganlawar A, Somani R. Protective effect of diosmin against diabetic neuropathy in experimental rats. J Integr Med, 2014; 12(1):35-41.

Joharchi K, Memari M, Azargashb E, Saadat N. Efficacy and safety of duloxetine and Pregabalin in Iranian patients with diabetic peripheral neuropathic pain: a double-blind, randomized clinical trial. Journal of Diabetes and Metabolic Disorders., 2019; 18(2):575-82.

Khwaja GA, Chaudhry N. Current and emerging therapies for painful diabetic neuropathies. J Indian Acad Clin Med, 2007; 8:53-64.

Kitto KF, Haley JE, Wilcox GL. Involvement of nitric oxide in spinally mediated hyperalgesia in the mouse. Neurosci Lett, 1992; 148(12):1-5.

Koltezenburg M, Scadding J. Neuropathic pain. Curr Opin Neurol, 2001; 14(5):641-7.

Lesser H, Sharma U, LaMoreaux L, Poole RM. Pregabalin relieves symptoms of painful diabetic neuropathy: a randomized controlled trial. Neurology, 2004; 63(11):2104-10.

Pradhan AD, Manson JE, Rifai N, Buring JE, Ridker PM. C-reactive protein, interleukin 6 , and risk of developing type 2 diabetes mellitus. JAMA, 2001; 286(3):327-34.

Ramirez MA, Borja NL. Epalrestat: an aldose reductase inhibitor for the treatment of diabetic neuropathy. Pharmacotherapy, 2008; 28(5):646-55.

Raskin J, Pritchett YL, Wang F, D'Souza DN, Waninger $\mathrm{AL}$, Iyengar S, Wernicke JF. A double-blind, randomized multicenter trial comparing duloxetine with placebo in the management of diabetic peripheral neuropathic pain. Pain Med, 2005; 6(5):346-56.

Richter RW, Portenoy R, Sharma U, Lamoreaux L, Bockbrader H, Knapp LE. Relief of painful diabetic peripheral neuropathy with pregabalin: a randomized, placebo-controlled trial. J Pain Res., 2005; 6(4):253-60.
Rosenstock J, Tuchman M, LaMoreaux L, Sharma U. Pregabalin for the treatment of painful diabetic peripheral neuropathy: a double-blind, placebo-controlled trial. Pain, 2004; 110(3):628-38.

Ryle C, Donaghy M. Non-enzymatic glycation of peripheral nerve proteins in human diabetics. J Neurol Sci, 1995; 129(1):62-8.

Singh GA, Bhardwaj S, Pandita D, Lather V, Singh Sekhon B. Updates on aldose reductase inhibitors for management of diabetic complications and non-diabetic diseases. Mini Reviews in Medicinal Chemistry., 2016; 16(2):120-62.

Sundaram M, Kavookjian J, Patrick JH, Miller LA, Madhavan SS, Scott VG. Quality of life, health status and clinical outcomes in Type 2 diabetes patients. Qual Life Res, 2007; 16(2):165-77.

Syed I. Glycated haemoglobin; past, present, and future are we ready for the change. Diabetes, 2011; 11:12.

Van Hecke O, Austin SK, Khan RA, Smith BH, Torrance N. Neuropathic pain in the general population: a systematic review of epidemiological studies. Pain., 2014; 155(4):654-62.

Veves A, Backonja M, Malik RA. Painful diabetic neuropathy: epidemiology, natural history, early diagnosis, and treatment options. Pain Med, 2008; 9:660-74.

Vinik A. The approach to the management of the patient with neuropathic pain. J Clin Endocrinol Metab, 2010; 95(11):4802-11.

Yagihashi S, Yamagishi SI, Wada R. Pathology and pathogenetic mechanisms of diabetic neuropathy: correlation with clinical signs and symptoms. Diabetes Res Clin Pract, 2007; 77(3):184-9.

Zychowska M, Rojewska E, Przewlocka B, Mika J. Mechanisms and pharmacology of diabetic neuropathy-experimental and clinical studies. Pharmacol Rep, 2013; 65(6):1601-10.

\section{How to cite this article:}

Singh R, Rao HK, Singh TG. Comparison of safety and efficacy of pregabalin, duloxetine and their combination with epalrestat in diabetic neuropathy: A prospective, doubleblind, randomized, controlled trial. J Appl Pharm Sci, 2021; 11 (Supp 1):071-079. 